Rev. Inst. Flor. v. 26 n. 1 p. $71-872014$

http://dx.doi.org/10.4322/rif.2014.005

ISSN impresso 0103-2674/on-line 2178-5031

\title{
ESTUDO FLORÍSTICO DE SEGMENTO DE MATA ATLÂNTICA EM PARELHEIROS, SÃO PAULO, SP, PARA SOLTURA DE BUGIO-RUIVO, Alouatta clamitans (Cabrera, 1940) ${ }^{1}$
}

\section{FLORISTIC SURVEY OF ATLANTIC RAIN FOREST IN THE DISTRICT OF PARELHEIROS, SÃO PAULO, SOUTHEASTERN BRAZIL, FOR REINTRODUCTION OF THE BROWN HOWLER MONKEY, Alouatta clamitans (Cabrera, 1940)}

\begin{abstract}
Ricardo José Francischetti GARCIA², ; Sumiko HONDA²; Brígida Gomes FRIES ${ }^{3}$
RESUMO - A fim de minimizar os efeitos da pressão da urbanização sobre bugios-ruivos, Alouatta clamitans (Cabrera, 1940), a Divisão de Fauna em parceria com o Herbário Municipal realiza, desde 1996, o Programa de Reintrodução de Bugios, com o objetivo de viabilizar o retorno à vida livre dos indivíduos aptos. Entre várias áreas prospectadas para possível soltura de bugios-ruivos, o Sítio Roda d'Água, distrito de Parelheiros, município de São Paulo, foi selecionado para realização do presente estudo. Todos os componentes da mata, em uma área de 89,9 hectares, foram amostrados para avaliação da disponibilidade de alimentos. Foram registradas 251 espécies vasculares, sendo 13 pteridófitas, uma gimnosperma e 237 angiospermas. O material testemunho está depositado no Herbário Municipal. Na área estudada foram registradas duas espécies ameaçadas e quatro quase ameaçadas. As espécies dos componentes arbóreo-arbustivo e epifítico, que constituem o hábitat preferencial dos bugios-ruivos, correspondem a $73,7 \%$ do total levantado. Dentro deste grupo de espécies, 17,8\% já foram registradas como fontes alimentares para a espécie-alvo. Dentre as espécies já referidas na dieta de bugios-ruivos, registradas na área de estudo, todas apresentam distribuição geográfica ampla. Desta forma, a área estudada foi considerada apropriada para a soltura de bugios-ruivos, quanto à disponibilidade de recursos para alimentação.
\end{abstract}

Palavras-chave: primatas; interação fauna-flora; conservação; reintrodução.

\begin{abstract}
In order to minimize the urbanization pressure effects over Brown Howler Monkeys, Alouatta clamitans (Cabrera, 1940), the Fauna Division in partnership with the Municipal Herbarium carries out a Brown Howler Monkey Reintroduction Program since 1996, with the aim of enabling the return of fit individuals to the wild. Among several areas prospected for possible reintroduction of the Brown Howler Monkey, Roda d'Água Ranch, District of Parelheiros, City of São Paulo, was selected to carry out this study. All components of forest, in an 89.9 hectares area, have been sampled for food availability assessment. Two hundred fifty-one vascular species, with 13 pteridophytes, 1 gymnosperm and 237 angiosperms were reported. The voucher specimen material was deposited in the Municipal Herbarium (PMSP). Two threatened species and four near-threatened species were found in the studied area. The species of arboreal-shrubby and epiphytic components that compose the preferred habitat of this primate species correspond to $73.7 \%$ of total surveyed. Within this species group, $17.8 \%$ have been recorded as food sources for the target species. Among the species already referred as Brown Howler Monkey diet, registered in the study area, all of them present a wide geographical distribution. Thereby, the study area was considered suitable for the Brown Howler Monkey release regarding to food resources availability.
\end{abstract}

Keywords: primates; fauna-flora interaction; conservation; reintroduction.

${ }_{1}^{1}$ Recebido para análise em 10.01.14. Aceito para publicação em 29.07.14.

${ }^{2}$ Prefeitura do Município de São Paulo, Secretaria Municipal do Verde e do Meio Ambiente, Herbário Municipal, Av. IV Centenário, 1286, Parque Ibirapuera, 04030-000 São Paulo, SP, Brasil.

${ }^{3}$ Prefeitura do Município de São Paulo, Secretaria Municipal do Verde e do Meio Ambiente, Divisão Técnica de Medicina Veterinária e Manejo da Fauna Silvestre, Av. IV Centenário, 1286, Parque Ibirapuera, 04030-000 São Paulo, SP, Brasil.

${ }^{4}$ Autor para correspondência: Ricardo José Francischetti Garcia - rfrancischetti@prefeitura.sp.gov.br 


\section{INTRODUÇÃO}

Alouatta clamitans (Cabrera, 1940), conhecido como bugio-ruivo, é um primata endêmico da Mata Atlântica, ameaçado de extinção segundo São Paulo (2014) e pouco preocupante segundo International Union for Conservation of Nature IUCN (2010). A distribuição da espécie estende-se da região de Missiones (Nordeste da Argentina) à vertente atlântica do Brasil, da região serrana central do Rio Grande do Sul a São Paulo, exceto nas suas regiões norte e nordeste, no litoral e porção norte do Rio de Janeiro e região do Rio Jequitinhonha em Minas Gerais (Gregorin, 2006).

A perda de hábitat decorrente da expansão urbana e as injúrias provocadas pela aproximação dos bugios-ruivos às residências (Fries et al., 2012) são alguns dos motivos de entrada destes animais na Divisão Técnica de Medicina Veterinária e Manejo da Fauna Silvestre (Divisão de Fauna) para tratamento.

A fim de minimizar os efeitos dessa pressão sobre a espécie, a Divisão de Fauna em parceria com o Herbário Municipal realiza, desde 1996, o Programa de Reintrodução de Bugios - PER, com o objetivo de viabilizar o retorno à vida livre dos indivíduos aptos, isto é, aqueles que apresentam estado clínico saudável e perfil comportamental adequado à espécie.

Em continuidade, o Conselho do Fundo Especial do Meio Ambiente e Desenvolvimento Sustentável - CONFEMA aprovou em 2006 o financiamento do Projeto "Manejo e conservação do bugio, Alouatta clamitans na Região Metropolitana de São Paulo: aprimorando o Programa de Reintrodução" (São Paulo, 2006), que incluiu os levantamentos florísticos nas áreas de soltura.

Com intuito de verificar a disponibilidade de recursos alimentares para manutenção dos indivíduos, análise recomendada pela Instrução Normativa $\mathrm{n}^{\mathrm{o}} 179$, de 25 de junho de 2008 (Instituto Brasileiro do Meio Ambiente e dos Recursos Naturais Renováveis - IBAMA, 2008) e pela União Internacional para Conservação da Natureza (Baker, 2002), levantamentos florísticos foram conduzidos.

Considerando que os recursos naturais existentes nos parques urbanos do município de São Paulo não preenchem as necessidades biológicas e ecológicas da espécie-alvo, conforme critérios de seleção elencados em Fries et al. (2012), realizou-se a prospecção de áreas apropriadas à soltura de bugios-ruivos.

Para este Projeto, as áreas foram previamente identificadas através de mapas, fotografias aéreas, visitas e entrevistas. Posteriormente, vistorias técnicas preliminares foram realizadas para verificar a possibilidade de promover solturas no local. Para a seleção de áreas foram considerados os pré-requisitos estabelecidos pela Divisão de Fauna, como tamanho da área do fragmento florestal, proximidade com áreas urbanas ou periurbanas, proximidade a estradas, existência de barreiras naturais ou construídas (como represas, rios, agricultura, pastagens), relação da população local com a fauna, ocorrência de grupos de bugios na região e/ou no local (Fries et al., 2012).

A partir dessas informações foram realizadas vistorias conjuntas das equipes do Herbário Municipal e da Divisão de Fauna aos locais pré-selecionados para avaliação da vegetação. Esta avaliação considerou a existência de estratificação (componentes arbóreo alto, arbóreo médio, arbustivo, herbáceo, epifítico e lianas); existência de bromélias-tanque (onde o acúmulo de água é um recurso importante para a dessedentação dos bugios-ruivos); formato do fragmento (buscando-se o menor efeito de borda possível); existência de espécies conhecidas como recursos alimentares para os bugios-ruivos.

Dentre as áreas vistoriadas, foi escolhido o Sítio Roda d'Água, em Parelheiros, município de São Paulo (Figura 1), para a realização do estudo florístico, com o objetivo de verificar se esta área possui recursos alimentares para os bugios-ruivos, aspecto fundamental para o sucesso da soltura.

\section{MATERIAL E MÉTODOS}

O Sítio Roda d'Água está localizado no município de São Paulo, distrito e subprefeitura de Parelheiros (2351'01'S, 465'53'W) apresentando área total de 118 ha, área de mata de 89,9 ha, altitudes entre $770 \mathrm{~m}$ e $822 \mathrm{~m}$. Na área sem cobertura florestal encontram-se área agricultável (hortaliças), pasto abandonado, brejo, lago, caminhos e construções rurais (estufas, casa dos sitiantes, depósitos). 


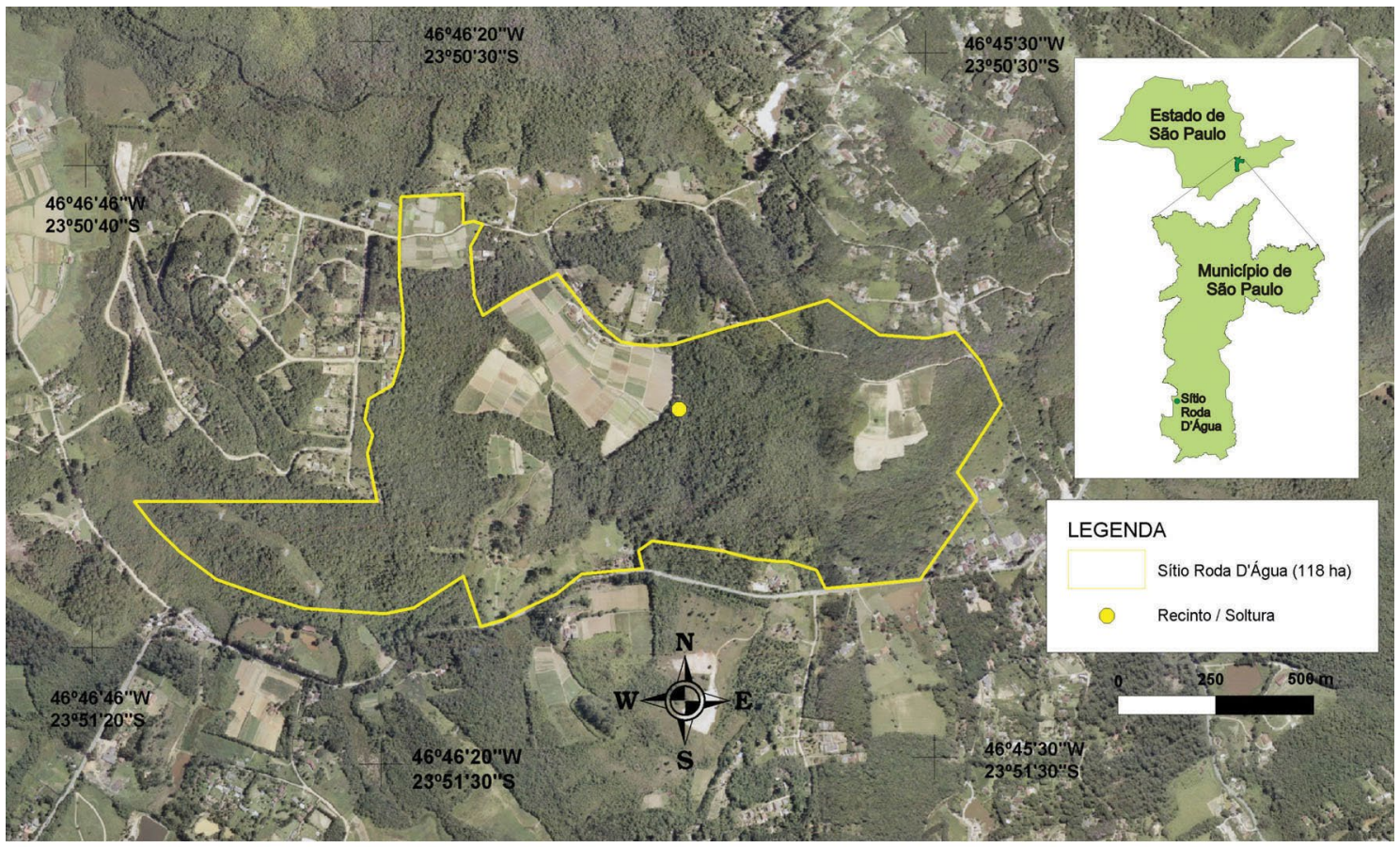

Figura 1. Mapa de localização da área de estudo, Sítio Roda d'Água, Parelheiros, São Paulo - SP, Brasil. (Fonte: SVMA/Divisão de Fauna).

Figure 1. Map of study area location, Roda d’Água Ranch, Parelheiros, São Paulo, State of São Paulo, Brazil. (Source: SVMA/Divisão de Fauna).

A área selecionada para estudo florístico foi visitada durante um ano, com coletas mensais. Foram percorridas as diversas trilhas, caminhos e bordas possíveis, coletando-se amostras de todos os componentes da vegetação.

Foram utilizados técnicas e materiais usuais de coleta (Fidalgo e Bononi, 1989). Para os exemplares arbóreos maiores que 15 metros, quando não foi possível realizar a coleta utilizando-se podão, foram organizadas coletas específicas em 29/11/2008, 06/12/2008 e 13/12/2008, com escalador (Biólogo Renato Schionato), que utilizou técnica e equipamento especializado para escalada (rapel).

Todo material coletado foi encaminhado ao Herbário Municipal para preparação de exsicatas e identificação segundo técnicas usuais (Fidalgo e Bononi, 1989). Materiais excedentes de coleta foram disponibilizados para a Divisão de Fauna para oferecimento esporádico e pontual aos bugios em cativeiro. Excedentes de epífitas também foram enviados à Divisão Técnica de Produção e Arborização (DEPAVE-2) para experimentação de cultivo.

A nomenclatura e a classificação adotadas estão de acordo com a Lista de Espécies da Flora do Brasil (Forzza et al., 2013). Pelo menos uma amostra de cada espécie registrada nas coletas foi incluída no acervo do Herbário Municipal (PMSP).

\section{RESULTADOS E DISCUSSÃO}

Foram realizadas 13 expedições de coleta entre outubro de 2007 e outubro de 2008, após a primeira visita de reconhecimento realizada em 25/04/2001.

Nesse período foram registradas 251 espécies de plantas vasculares, sendo 237 espécies pertencentes a 71 famílias de angiospermas, uma espécie de uma família de gimnosperma e 13 espécies de 7 famílias de pteridófitas (Tabela 1). 
Tabela 1. Levantamento florístico do Sítio Roda d’Água, Parelheiros, São Paulo, SP. Voucher: G: R.J.F. Garcia; GG: G. Garbin; H: S. Honda; S: R. Schionato; *: observações de campo. Hábito: arb: arbusto; árv: árvore (> $4 \mathrm{~m}$ alt.); avt: arvoreta (até $4 \mathrm{~m}$ alt.); b: bambusoides; eces: erva cespitosa; ee: erva ereta; ep: epífita; epr: erva prostrada/ rastejante; eriz: erva rizomatosa; eros: erva rosulada; fa: feto arborescente; hp: hemiparasita; pal: palmeira; tr: trepadeira. Distribuição geográfica (Forzza et al., 2013): EBR: brasileira e extrabrasileira; BRA: endêmica do Brasil em Mata Atlântica e outros domínios; MAT: endêmica de Mata Atlântica, SPE: endêmica do Estado de São Paulo; ND: dado não disponível. Estado de Conservação (Mamede et al., 2007): NT: quase ameaçada, VU: vulnerável. PEFI: ocorrência no Parque Estadual das Fontes do Ipiranga. Alimento: 1: Carvalho (1975), 2: Chitolina e Sander (1981), 3: Galetti et al. (1994), 4: Kuhlmann (1975), 5: Miranda e Passos (2004), 6: Silva Jr. (1980), 7: Vasconcellos e Aguiar (1982); fo: folha; fr: fruto; s: semente; D: pode ser usada para dessedentação.

Table 1. Floristic inventory of Roda d'Água Ranch, Parelheiros, São Paulo, SP. Voucher: G: R.J.F. Garcia; GG: G. Garbin; H: S. Honda; S: R. Schionato; *: Field observations. Habit: arb: shrub; árv: tree ( $>4 \mathrm{~m}$ alt.); avt: small tree $(<4 \mathrm{~m}$ alt.); b: bamboos; eces: cespitose herb; ee: erect herb; ep: epiphyte; epr: prostrate/creeping herb; eriz: rhizomatous herb; eros: rosulate herb; fa: arborescent fern; hp: hemiparasite; pal: palm tree; tr: climbing plant. Geografic distribution (Forzza et al., 2013): EBR: Brazilian and extra-Brazilian; BRA: endemic to Brazil in Atlantic Rain Forest and other phytogeografical regions; MAT: endemic to Atlantic Rain Forest, SPE: endemic to the State of São Paulo; ND: data not available. Conservation Status (Mamede et al., 2007): NT: near threatened, VU: vulnerable. PEFI: ocurrency in Parque Estadual das Fontes do Ipiranga. Food: 1: Carvalho (1975), 2: Chitolina e Sander (1981), 3: Galetti et al. (1994), 4: Kuhlmann (1975), 5: Miranda e Passos (2004), 6: Silva Jr. (1980), 7: Vasconcellos e Aguiar (1982); fo: leaf; fr: fruit; s: seed; D: can be used to quench the thirst.

\begin{tabular}{llllll}
\hline Grupo & Voucher hábito & Distribuição & Estado de & PEFI alimento \\
Família & de & & geográfica & Conservação & \\
Espécie & coleta & & & & \\
\hline
\end{tabular}

PTERIDÓFITAS

ANEMIACEAE

Anemia phyllitidis (L.) Sw. $\quad$ H 411 eces $\quad$ EBR

ASPLENIACEAE

Asplenium mucronatum C.Presl

H 496 ep EBR

BLECHNACEAE

Blechnum brasiliense Desv.

$\begin{array}{llll}\text { H } 389 & \text { eros } & \text { EBR } & \text { X } \\ \text { H 694 } & \text { tr } & \text { EBR } & \text { X }\end{array}$

Salpichlaena volubilis (Kaulf.) J.Sm.

CYATHEACEAE

Cyathea atrovirens (Langsd. \& Fisch.) Domin $\quad$ H 806 fa $\quad$ MA

DRYOPTERIDACEAE

Ctenitis sp.

H 401 eces ND

Lastreopsis amplissima (C.Presl) Tindale

H 656 ep EBR

X

GLEICHENIACEAE

Sticherus bifidus (Willd.) Ching

H 727 eriz

POLYPODIACEAE

Campyloneurum repens (Aubl.) C.Presl

Microgramma squamulosa (Kaulf.) de la Sota

H 691 ep EBR

Pleopeltis hirsutissima (Raddi) de la Sota

H 800 ep EBR

Pleopeltis pleopeltifolia (Raddi) Alston

H 780 ep EBR

Serpocaulon catharinae (Langsd. \& Fisch.) A.R.Sm.

H 501 ep MAT

H 497 ep MAT

continua

to be continued 
GARCIA, R.J.F.; HONDA, S.; FRIES, B.G. Estudo florístico de Mata Atlântica para soltura de bugio-ruivo, Alouatta clamitans

continuação - Tabela 1

continuation - Table 1

\begin{tabular}{lllll}
\hline Grupo & $\begin{array}{l}\text { Voucher hábito } \\
\text { Família }\end{array}$ & $\begin{array}{l}\text { Distribuição } \\
\text { de Estado de } \\
\text { Espécie }\end{array}$ & coleta & PEFI alimento \\
Conservação & & \\
\hline
\end{tabular}

GIMNOSPERMAS

ARAUCARIACEAE

Araucaria angustifolia (Bertol.) Kuntze

$\begin{array}{llll}\text { H } 688 \text { árv } & \text { EBR } \quad \text { VU } \quad \begin{array}{l}1(\mathrm{~s}), 2(\mathrm{bf}) \\ 5(\mathrm{~s})\end{array}\end{array}$

\section{ANGIOSPERMAS}

\section{ACANTHACEAE}

Mendoncia puberula Mart.

\begin{tabular}{|c|c|c|c|c|c|}
\hline Н 618 & $\operatorname{tr}$ & BRA & & $X$ & \\
\hline Н 328 & $\operatorname{tr}$ & BRA & & $\mathrm{X}$ & 4 (fr) \\
\hline Н 393 & árv & EBR & & $\mathrm{X}$ & 5 (fo, fr) \\
\hline G 3206 & árv & MAT & & $X$ & 4 (fr), 5 (fr) \\
\hline G 3451 & avt & EBR & & & \\
\hline Н 357 & ee & EBR & & & \\
\hline S 26 & árv & ND & & & \\
\hline G 3201 & ep & MAT & & $X$ & \\
\hline Н 350 & $\operatorname{tr}$ & MAT & & $X$ & \\
\hline$*$ & pal & ND & & & \\
\hline Н 649 & pal & MAT & NT & $\mathrm{X}$ & \\
\hline Н 511 & pal & EBR & & $X$ & $\begin{array}{l}2(\mathrm{fr}), \\
3(\mathrm{fr}), 5(\mathrm{fr})\end{array}$ \\
\hline
\end{tabular}

\section{ASTERACEAE}

Baccharis anomala DC.

$\begin{array}{lll}\text { H 716 } & \text { arb } & \text { EBR } \\ \text { H 391 } & \text { tr } & \text { MAT } \\ \text { H 621 } & \text { ee } & \text { BRA } \\ \text { H 623 } & \text { árv } & \text { BRA } \\ \text { G 3208 } & \text { avt } & \text { BRA } \\ \text { H 714 } & \text { avt } & \text { ND } \\ \text { G 3204 } & \text { tr } & \text { EBR } \\ \text { H 413 } & \text { ee } & \text { EBR } \\ \text { H 699 } & \text { epr } & \text { BRA } \\ \text { H 467 } & \text { ee } & \text { EBR } \\ \text { H 384 } & \text { ee } & \text { EBR }\end{array}$

$X$
$X$
$X$
$X$
$X$
$X$
$X$
$X$
$X$

Erechtites valerianiifolius (Wolf) DC.

H 384 ee EBR 
continuação - Tabela 1

continuation - Table 1

\begin{tabular}{|c|c|c|c|c|c|c|}
\hline $\begin{array}{l}\text { Grupo } \\
\text { Família } \\
\text { Espécie }\end{array}$ & $\begin{array}{l}\text { Voucher } \\
\text { de } \\
\text { coleta }\end{array}$ & hábito & $\begin{array}{l}\text { Distribuição } \\
\text { geográfica }\end{array}$ & $\begin{array}{l}\text { Estado de } \\
\text { Conservação }\end{array}$ & PEFI & alimento \\
\hline Gochnatia sp. & H 705 & arb & ND & & & \\
\hline Grazielia intermedia (DC.) R.M.King \& H.Rob. & Н 394 & ee & EBR & & $\mathrm{X}$ & \\
\hline Heterocondylus alatus (Vell.) R.M.King \& H.Rob. & Н 733 & arb & BRA & & $\mathrm{X}$ & \\
\hline Lactuca sp. & Н 316 & ee & ND & & & \\
\hline Lepidaploa $\mathrm{sp}$. & H 704 & arb & ND & & & \\
\hline Leptostelma maxima D.Don & Н 387 & ee & EBR & & $\mathrm{X}$ & \\
\hline Mikania hirsutissima DC. & H 701 & $\operatorname{tr}$ & EBR & & $\mathrm{X}$ & \\
\hline Mikania micrantha Kunth & H 601 & $\operatorname{tr}$ & EBR & & $\mathrm{X}$ & \\
\hline Mikania sericea Hook. \& Arn. & H 628 & $\operatorname{tr}$ & MAT & & & \\
\hline Mikania ternata (Vell.) B.L.Rob. & H 625 & $\operatorname{tr}$ & EBR & & & \\
\hline Piptocarpha axillaris (Less.) Baker & H 771 & árv & MAT & & $\mathrm{X}$ & \\
\hline Piptocarpha macropoda (DC.) Baker & H 787 & árv & MAT & & & $\begin{array}{l}1 \text { (fo), } 4 \text { (bf, } \\
\text { fo), } 7 \text { (fo) }\end{array}$ \\
\hline Senecio sp. & Н 726 & ee & ND & & & \\
\hline Solidago chilensis Meyen & H 510 & ee & EBR & & $\mathrm{X}$ & \\
\hline Vernonanthura divaricata (Spreng.) H.Rob. & G 3192 & árv & EBR & & $\mathrm{X}$ & $\begin{array}{l}1 \text { (fo), } \\
4 \text { (bf, fo), } \\
7 \text { (fo) }\end{array}$ \\
\hline Vernonanthura sp. & H 789 & arb & ND & & & \\
\hline BEGONIACEAE & & & & & & \\
\hline $\begin{array}{l}\text { Begonia cucullata Willd. var. cucullata } \\
\text { BIGNONIACEAE }\end{array}$ & Н 382 & ee & EBR & & $\mathrm{X}$ & \\
\hline Jacaranda puberula Cham. & H 611 & árv & MAT & & $\mathrm{X}$ & \\
\hline Pyrostegia venusta (Ker Gawl.) Miers & Н 709 & $\operatorname{tr}$ & EBR & & $\mathrm{X}$ & $3(\mathrm{fl})$ \\
\hline n.i. & Н 796 & $\operatorname{tr}$ & ND & & & \\
\hline BORAGINACEAE & & & & & & \\
\hline Varronia guazumaefolia Desv. & H 813 & arb & BRA & & & \\
\hline Varronia polycephala Lam. & H 334 & arb & EBR & & & \\
\hline BRASSICACEAE & & & & & & \\
\hline Brassica juncea (L.) Czern. & H 604 & ee & EBR & & & \\
\hline BROMELIACEAE & & & & & & \\
\hline Aechmea cylindrata Lindm. & G 3252 & eros & MAT & & & $\mathrm{D}$ \\
\hline Tillandsia stricta Sol. & Н 696 & ep & BRA & & $\mathrm{X}$ & \\
\hline Tillandsia tenuifolia $\mathrm{L}$. & Н 775 & ep & BRA & & $\mathrm{X}$ & \\
\hline Vriesea carinata Wawra var. carinata & Н 661 & eros & MAT & & $\mathrm{X}$ & $\mathrm{D}$ \\
\hline Vriesea friburgensis $\mathrm{Mez}$ & $*$ & ep & BRA & & $\mathrm{X}$ & $\mathrm{D}$ \\
\hline Vriesea heterostachys (Baker) L.B.Sm. & H 502 & ep & MAT & & $\mathrm{X}$ & $\mathrm{D}$ \\
\hline
\end{tabular}

continua to be continued 
GARCIA, R.J.F.; HONDA, S.; FRIES, B.G. Estudo florístico de Mata Atlântica para soltura de bugio-ruivo, Alouatta clamitans

continuação - Tabela 1

continuation - Table 1

\begin{tabular}{|c|c|c|c|c|c|c|}
\hline $\begin{array}{l}\text { Grupo } \\
\text { Família } \\
\text { Espécie }\end{array}$ & $\begin{array}{l}\text { Voucher } \\
\text { de } \\
\text { coleta }\end{array}$ & hábito & $\begin{array}{l}\text { Distribuição } \\
\text { geográfica }\end{array}$ & $\begin{array}{l}\text { Estado de } \\
\text { Conservação }\end{array}$ & PEFI & alimento \\
\hline Vriesea incurvata Gaudich. & H 498 & ep & MAT & & $\mathrm{X}$ & $\mathrm{D}$ \\
\hline $\begin{array}{l}\text { Wittrockia cyathiformis (Vell.) Leme } \\
\text { CACTACEAE }\end{array}$ & Н 659 & ep & MAT & & & $\mathrm{D}$ \\
\hline Hatiora salicornioides (Haw.) Britton \& Rose & H 802 & ep & MAT & & $\mathrm{X}$ & \\
\hline Lepismium houlletianum (Lem.) Barthlott & H 651 & ep & EBR & & $\mathrm{X}$ & \\
\hline $\begin{array}{l}\text { Rhipsalis burchellii Britton \& Rose } \\
\text { CAMPANULACEAE }\end{array}$ & Н 332 & ep & MAT & & $\mathrm{X}$ & \\
\hline $\begin{array}{l}\text { Lobelia exaltata Pohl } \\
\text { CANNABACEAE }\end{array}$ & H 385 & ee & MAT & & $\mathrm{X}$ & \\
\hline $\begin{array}{l}\text { Trema micrantha (L.) Blume } \\
\text { CARDIOPTERIDACEAE }\end{array}$ & Н 354 & árv & EBR & & $\mathrm{X}$ & \\
\hline $\begin{array}{l}\text { Citronella paniculata (Mart.) R.A.Howard } \\
\text { CLETHRACEAE }\end{array}$ & G 3211 & árv & EBR & & $\mathrm{X}$ & \\
\hline $\begin{array}{l}\text { Clethra scabra Pers. } \\
\text { COMMELINACEAE }\end{array}$ & Н 338 & árv & EBR & & $\mathrm{X}$ & 5 (fo) \\
\hline $\begin{array}{l}\text { Tripogandra diuretica (Mart.) Handlos } \\
\text { CONVOLVULACEAE }\end{array}$ & G 3247 & eriz & BRA & & $\mathrm{X}$ & \\
\hline $\begin{array}{l}\text { Ipomoea indica (Burm.) Merrill } \\
\text { CUCURBITACEAE }\end{array}$ & G 3215 & $\operatorname{tr}$ & EBR & & $\mathrm{X}$ & \\
\hline $\begin{array}{l}\text { Wilbrandia hibiscoides Silva Manso } \\
\text { CYPERACEAE }\end{array}$ & Н 403 & $\operatorname{tr}$ & BRA & & & \\
\hline Cyperus cf. meyenianus Kunth & H 512 & eces & ND & & & \\
\hline Pleurostachys foliosa Kunth & Н 349 & ee & MAT & & $\mathrm{X}$ & \\
\hline $\begin{array}{l}\text { Scleria latifolia Kunth } \\
\text { DILLENIACEAE }\end{array}$ & H 818 & ee & EBR & & $\mathrm{X}$ & \\
\hline Scleria panicoides Kunth & H 615 & ee & EBR & & $\mathrm{X}$ & \\
\hline $\begin{array}{l}\text { Davilla rugosa Poir. } \\
\text { ELAEOCARPACEAE }\end{array}$ & Н 713 & $\operatorname{tr}$ & EBR & & $\mathrm{X}$ & \\
\hline $\begin{array}{l}\text { Sloanea hirsuta (Schott) Planch. ex Benth. } \\
\text { EUPHORBIACEAE }\end{array}$ & Н 397 & avt & MAT & & $\mathrm{X}$ & \\
\hline Alchornea sidifolia Müll.Arg. & G 3209 & árv & EBR & & $\mathrm{X}$ & 7 (fo) \\
\hline Croton celtidifolius Baill. & H 355 & avt & MAT & & & \\
\hline Dalechampia triphylla Lam. & Н 619 & $\operatorname{tr}$ & BRA & & $\mathrm{X}$ & \\
\hline Ricinus communis $\mathrm{L}$. & H 335 & arb & EBR & & $\mathrm{X}$ & \\
\hline $\begin{array}{l}\text { Sapium sellowianum (Müll.Arg.) Klotzsch ex Baill. } \\
\text { FABACEAE }\end{array}$ & H 406 & árv & MAT & & & \\
\hline Dalbergia brasiliensis Vogel & Н 386 & árv & BRA & & $\mathrm{X}$ & \\
\hline
\end{tabular}

to be continued 
continuação - Tabela 1

continuation - Table 1

\begin{tabular}{|c|c|c|c|c|c|c|}
\hline $\begin{array}{l}\text { Grupo } \\
\text { Família } \\
\text { Espécie } \\
\end{array}$ & $\begin{array}{l}\text { Voucher } \\
\text { de } \\
\text { coleta }\end{array}$ & hábito & $\begin{array}{l}\text { Distribuição } \\
\text { geográfica }\end{array}$ & $\begin{array}{l}\text { Estado de } \\
\text { Conservação }\end{array}$ & PEFI & alimento \\
\hline Dalbergia frutescens (Vell.) Britton & S 20 & tr/árv & EBR & & $\mathrm{X}$ & 3 (fo) \\
\hline Desmodium affine Schltdl. & Н 358 & epr & EBR & & $\mathrm{X}$ & \\
\hline Hymenaea courbaril $\mathrm{L}$. & H 359 & árv & EBR & & $\mathrm{X}$ & 3 (fo) \\
\hline Inga barbata Benth. & H 620 & arb & BRA & & & \\
\hline Inga sellowiana Benth. & S 43 & árv & MAT & & $\mathrm{X}$ & $4(\mathrm{fr}, \mathrm{s})$ \\
\hline Inga sessilis (Vell.) Mart. & G 3202 & árv & BRA & & $\mathrm{X}$ & 7 (fo, fr) \\
\hline Machaerium hirtum (Vell.) Stellfeld & H 402 & árv & EBR & & $\mathrm{X}$ & \\
\hline Phanera microstachya (Raddi) L.P.Queiroz & H 735 & $\operatorname{tr}$ & EBR & & & \\
\hline Piptadenia adiantoides (Spreng.) J.F.Macbr. & H 731 & $\operatorname{tr}$ & BRA & & & \\
\hline Senegalia sp. & H 380 & $\operatorname{tr}$ & ND & & & \\
\hline Senna multijuga (Rich.) H.S.Irwin \& Barneby & H 412 & árv & EBR & & $\mathrm{X}$ & \\
\hline Tachigali denudata (Vogel) Oliveira-Filho & S 50 & árv & MAT & & $\mathrm{X}$ & \\
\hline Vicia sativa $\mathrm{L}$. & Н 793 & epr & EBR & & & \\
\hline \multicolumn{7}{|l|}{ GESNERIACEAE } \\
\hline Nematanthus villosus (Hanst.) Wieher & H 655 & ep & SPE & & $\mathrm{X}$ & \\
\hline \multicolumn{7}{|l|}{ IRIDACEAE } \\
\hline Sisyrinchium vaginatum Spreng. & H 614 & ee & EBR & NT & & \\
\hline \multicolumn{7}{|l|}{ LAMIACEAE } \\
\hline Hyptis lacustris A.St.-Hil. ex Benth. & H 466 & ee & EBR & & & \\
\hline Hyptis multibracteata Benth. & Н 390 & ee & MAT & & $\mathrm{X}$ & \\
\hline Hyptis sidifolia (L'Hér.) Briq. & H 468 & ee & EBR & & $\mathrm{X}$ & \\
\hline Leonurus japonicus Houtt. & H 794 & ee & EBR & & & \\
\hline Vitex polygama Cham. & Н 323 & árv & BRA & & $\mathrm{X}$ & \\
\hline \multicolumn{7}{|l|}{ LAURACEAE } \\
\hline Endlicheria paniculata (Spreng.) J.F. Macbr. & H 804 & arb & EBR & & $\mathrm{X}$ & \\
\hline Ocotea glaziovii Mez & H 469 A & árv & BRA & & & \\
\hline Ocotea pulchra Vattimo-Gil & Н 398 & árv & MAT & & $\mathrm{X}$ & \\
\hline Ocotea teleiandra (Meisn.) Mez & H 658 & árv, avt & MAT & & & \\
\hline \multicolumn{7}{|l|}{ LECYTHIDACEAE } \\
\hline Cariniana estrellensis (Raddi) Kuntze & $*$ & árv & EBR & & $\mathrm{X}$ & 3 (fo), 6 (fo) \\
\hline \multicolumn{7}{|l|}{ LOGANIACEAE } \\
\hline Strychnos sp. & H 695 & $\operatorname{tr}$ & ND & & & \\
\hline
\end{tabular}

continua to be continued 
GARCIA, R.J.F.; HONDA, S.; FRIES, B.G. Estudo florístico de Mata Atlântica para soltura de bugio-ruivo, Alouatta clamitans

continuação - Tabela 1

continuation - Table 1

\begin{tabular}{|c|c|c|c|c|c|c|}
\hline $\begin{array}{l}\text { Grupo } \\
\text { Família } \\
\text { Espécie } \\
\end{array}$ & $\begin{array}{l}\text { Voucher } \\
\text { de } \\
\text { coleta }\end{array}$ & hábito & $\begin{array}{l}\text { Distribuição } \\
\text { geográfica }\end{array}$ & $\begin{array}{l}\text { Estado de } \\
\text { Conservação }\end{array}$ & PEFI & alimento \\
\hline \multicolumn{7}{|l|}{ LYTHRACEAE } \\
\hline $\begin{array}{l}\text { Cuphea calophylla Cham. \& Schltdl. subsp. } \\
\text { mesostemon (Koehne) Lourteig }\end{array}$ & H 728 & epr & BRA & & $\mathrm{X}$ & \\
\hline \multicolumn{6}{|l|}{ MAGNOLIACEAE } & \\
\hline \multicolumn{6}{|l|}{ MALPIGHIACEAE } & \\
\hline Byrsonima ligustrifolia A.Juss. & G 3237 & árv & MAT & & $\mathrm{X}$ & $4(\mathrm{fr})$ \\
\hline \multicolumn{7}{|l|}{ MALVACEAE } \\
\hline Pseudobombax grandiflorum (Cav.) A. Robyns & G 3214 & árv & BRA & & $\mathrm{X}$ & \\
\hline Sida santaremensis Monteiro & H 405 & arb & EBR & & & \\
\hline \multicolumn{7}{|l|}{ MARANTACEAE } \\
\hline Calathea monophylla Körn. & Н 504 & eces & MAT & & & \\
\hline Ctenanthe lanceolata Petersen & Н 343 & eces & MAT & & $\mathrm{X}$ & \\
\hline \multicolumn{7}{|l|}{ MELASTOMATACEAE } \\
\hline Bertolonia mosenii Cogn. & Н 506 & ee & MAT & & & \\
\hline Leandra amplexicaulis DC. & Н 340 & arb & MAT & & $\mathrm{X}$ & \\
\hline Leandra australis (Cham.) Cogn. & H 720 & arb & EBR & & $\mathrm{X}$ & \\
\hline Leandra dasytricha (A.Gray) Cogn. & H 400 & arb & MAT & & $\mathrm{X}$ & \\
\hline Leandra melastomoides Raddi & G 3240 & arb & EBR & & $\mathrm{X}$ & \\
\hline Leandra refracta Cogn. & Н 341 & arb & MAT & & $\mathrm{X}$ & \\
\hline Miconia cabucu Hoehne & Н 807 & árv & MAT & & $\mathrm{X}$ & $4(s)$ \\
\hline Miconia fasciculata Gardner & G 3445A & árv & MAT & & & \\
\hline Miconia inconspicua Miq. & Н 786 & avt & MAT & & $\mathrm{X}$ & \\
\hline Miconia petropolitana Cogn. & H 722 & arb & MAT & & $\mathrm{X}$ & \\
\hline Miconia sellowiana Naudin & Н 791 & árv & BRA & & $\mathrm{X}$ & \\
\hline Tibouchina cerastifolia Cogn. & Н 407 & ee & BRA & & $\mathrm{X}$ & \\
\hline Tibouchina pulchra Cogn. & G 3246 & árv & MAT & & $\mathrm{X}$ & \\
\hline Tibouchina sellowiana Cogn. & H 513 & avt & MAT & & $\mathrm{X}$ & \\
\hline \multicolumn{7}{|l|}{ MELIACEAE } \\
\hline Cabralea canjerana (Vell.) Mart. & Н 801 & árv & BRA & & $\mathrm{X}$ & 3 (fo), 6 (fo) \\
\hline Cedrela fissilis Vell. & Н 797 & árv & EBR & & $\mathrm{X}$ & $\begin{array}{l}5(\mathrm{fo}), 6(\mathrm{fo}) \\
7 \text { (bf) }\end{array}$ \\
\hline Guarea macrophylla Vahl & H 650 & avt & EBR & & $\mathrm{X}$ & \\
\hline
\end{tabular}

continua

to be continued 
continuação - Tabela 1

continuation - Table 1

\begin{tabular}{|c|c|c|c|c|c|c|}
\hline $\begin{array}{l}\text { Grupo } \\
\text { Família } \\
\text { Espécie }\end{array}$ & $\begin{array}{l}\text { Voucher } \\
\text { de } \\
\text { coleta }\end{array}$ & hábito & $\begin{array}{l}\text { Distribuição } \\
\text { geográfica }\end{array}$ & $\begin{array}{l}\text { Estado de } \\
\text { Conservação }\end{array}$ & PEFI & alimento \\
\hline \multicolumn{7}{|l|}{ MENISPERMACEAE } \\
\hline \multicolumn{7}{|l|}{ MONIMIACEAE } \\
\hline Mollinedia schottiana (Spreng.) Perkins & Н 603 & arb & MAT & & $\mathrm{X}$ & \\
\hline Mollinedia uleana Perkins & H 777 & avt & MAT & & $\mathrm{X}$ & \\
\hline \multicolumn{7}{|l|}{ MORACEAE } \\
\hline Dorstenia hirta Desv. & G 3241 & ee & MAT & & & \\
\hline \multicolumn{7}{|l|}{ MYRTACEAE } \\
\hline Calyptranthes grandifolia O. Berg & H 409 & avt & MAT & & $\mathrm{X}$ & \\
\hline Campomanesia guaviroba (DC.) Kiaersk. & H 692 & árv & EBR & & $\mathrm{X}$ & \\
\hline Campomanesia phaea (O. Berg) Landrum & G 3440 & árv & MAT & NT & $\mathrm{X}$ & \\
\hline Eugenia cerasiflora Miq. & H 776 & árv & BRA & & $\mathrm{X}$ & \\
\hline Eugenia dodonaeifolia Cambess. & Н 364 & árv & MAT & & $\mathrm{X}$ & \\
\hline Marlierea suaveolens Cambess. & G 3438 & avt & MAT & $\mathrm{VU}$ & & \\
\hline Myrcia anacardiifolia Gardner & H 654 & árv & MAT & & $\mathrm{X}$ & \\
\hline \multicolumn{7}{|l|}{ NYCTAGINACEAE } \\
\hline Guapira nitida (Mart. ex J.A. Schmidt) Lundell & G 3245 & árv & MAT & & & \\
\hline $\begin{array}{l}\text { Guapira opposita (Vell.) Reitz } \\
\text { OCHNACEAE }\end{array}$ & H 707 & avt & EBR & & $\mathrm{X}$ & $4(s)$ \\
\hline $\begin{array}{l}\text { Ouratea parviflora (DC.) Baill. } \\
\text { OLACACEAE }\end{array}$ & \multicolumn{5}{|c|}{ OLACACEAE } & \\
\hline $\begin{array}{l}\text { Heisteria silvianii Schwacke } \\
\text { ORCHIDACEAE }\end{array}$ & H 808 & árv & BRA & & $\mathrm{X}$ & \\
\hline Gomesa crispa (Lindl.) Klotzsch ex Rchb.f. & Н 351 & ep & EBR & & $\mathrm{X}$ & \\
\hline n.i. sp.1 & G 3253 & ep & ND & & & \\
\hline n.i. sp. 2 & H 613 & ee & ND & & & \\
\hline \multicolumn{7}{|l|}{ PERACEAE } \\
\hline \multicolumn{7}{|l|}{ PHRYMACEAE } \\
\hline Mazus japonicus (Thunb.) Kuntze & H 342 & ee & EBR & & & \\
\hline \multicolumn{7}{|l|}{ PICRAMNIACEAE } \\
\hline $\begin{array}{l}\text { Picramnia ciliata Mart. } \\
\text { PIPERACEAE }\end{array}$ & \multicolumn{5}{|c|}{ PIPERACEAE } & \\
\hline Peperomia catharinae Miq. & Н 629 & ep & EBR & & $\mathrm{X}$ & \\
\hline
\end{tabular}

continua to be continued 
GARCIA, R.J.F.; HONDA, S.; FRIES, B.G. Estudo florístico de Mata Atlântica para soltura de bugio-ruivo, Alouatta clamitans

continuação - Tabela 1

continuation - Table 1

\begin{tabular}{|c|c|c|c|c|c|c|}
\hline $\begin{array}{l}\text { Grupo } \\
\text { Família } \\
\text { Espécie } \\
\end{array}$ & $\begin{array}{l}\text { Voucher } \\
\text { de } \\
\text { coleta }\end{array}$ & hábito & $\begin{array}{l}\text { Distribuição } \\
\text { geográfica }\end{array}$ & $\begin{array}{l}\text { Estado de } \\
\text { Conservação }\end{array}$ & PEFI & alimento \\
\hline Peperomia tetraphylla (G.Forst.) Hook. \& Arn. & H 781 & ep & EBR & & $\mathrm{X}$ & \\
\hline Piper caldense C.DC. & H 602 & arb & BRA & & $\mathrm{X}$ & \\
\hline Piper gaudichaudianum Kunth & H 774 & arb & BRA & & $\mathrm{X}$ & \\
\hline Piper mollicomum Kunth & Н 399 & arb & BRA & & $\mathrm{X}$ & \\
\hline \multicolumn{7}{|l|}{ PLANTAGINACEAE } \\
\hline Plantago australis Lam. & Н 346 & eros & EBR & & $\mathrm{X}$ & \\
\hline Veronica persica Poir. & H 606 & epr & EBR & & & \\
\hline \multicolumn{7}{|l|}{ POACEAE } \\
\hline Chusquea bambusoides (Raddi) Hack. & G 3200 & $\mathrm{~b}$ & MAT & & & \\
\hline Dichanthelium hebotes (Trin.) Zuloaga & Н 324 & eces & EBR & & & \\
\hline Ichnanthus leiocarpus (Spreng.) Kunth & H 352 & eces & EBR & & $\mathrm{X}$ & \\
\hline Ichnanthus pallens (Sw.) Munro ex Kunth & H 507 & epr & EBR & & $\mathrm{X}$ & \\
\hline Ichnanthus ruprechtii Döll & G 3439 & eriz & EBR & & & \\
\hline Lasiasis divaricata (L.) Hitchc. & H 514 & $\mathrm{~b}$ & EBR & & $\mathrm{X}$ & \\
\hline Olyra glaberrima Raddi & G 3239 & eriz & EBR & & $\mathrm{X}$ & \\
\hline Paspalum corcovadense Raddi & Н 325 & epr & EBR & & & \\
\hline Paspalum urvillei Steud. & GG 15 & eces & EBR & & $\mathrm{X}$ & \\
\hline Pharus lappulaceus Aubl. & G 3442 & eces & EBR & & $\mathrm{X}$ & \\
\hline Setaria sulcata Raddi & Н 509 & eces & EBR & & $\mathrm{X}$ & \\
\hline Sporobolus indicus (L.) R. Br. & Н 317 & eces & EBR & & & \\
\hline $\begin{array}{l}\text { Urochloa brizantha (Hochst. ex A.Rich.) Webster } \\
\text { POLYGALACEAE }\end{array}$ & Н 318 & eces & EBR & & $\mathrm{X}$ & \\
\hline Diclidanthera laurifolia Mart. & S 25 & $\operatorname{tr}$ & BRA & & $\mathrm{X}$ & $4(s)$ \\
\hline $\begin{array}{l}\text { Polygala lancifolia A.St.-Hil. \& Moq. } \\
\text { POLYGONACEAE }\end{array}$ & H 725 & ee & EBR & & $\mathrm{X}$ & \\
\hline Coccoloba warmingii Meisn. & H 388 & árv & BRA & & $\mathrm{X}$ & \\
\hline PRIMULACEAE & & & & & & \\
\hline $\begin{array}{l}\text { Myrsine umbellata Mart. } \\
\text { ROSACEAE }\end{array}$ & H 647 & árv & EBR & & $\mathrm{X}$ & 4 (fr) \\
\hline Prunus myrtifolia (L.) Urb. & G 2073 & árv & EBR & & $X$ & $\begin{array}{l}4 \text { (fr), } 5 \text { (fr), } \\
7 \text { (fr) }\end{array}$ \\
\hline
\end{tabular}


continuação - Tabela 1

continuation - Table 1

\begin{tabular}{|c|c|c|c|c|c|c|}
\hline $\begin{array}{l}\text { Grupo } \\
\text { Família } \\
\text { Espécie }\end{array}$ & $\begin{array}{l}\text { Voucher } \\
\text { de } \\
\text { coleta }\end{array}$ & hábito & $\begin{array}{l}\text { Distribuição } \\
\text { geográfica }\end{array}$ & $\begin{array}{l}\text { Estado de } \\
\text { Conservação }\end{array}$ & PEFI & alimento \\
\hline Rubus rosifolius $\mathrm{Sm}$. & H 712 & ee & EBR & & $\mathrm{X}$ & \\
\hline Rubus urticifolius Poir. & H 383 & arb & EBR & & & \\
\hline \multicolumn{7}{|l|}{ RUBIACEAE } \\
\hline Alseis floribunda Schott & H 322 & árv & BRA & & & \\
\hline Bathysa australis (A. St.-Hil.) K. Schum. & H 315 & árv & MAT & & $\mathrm{X}$ & \\
\hline Borreria latifolia (Aubl.) K.Schum. & H 330 & epr & EBR & & $\mathrm{X}$ & \\
\hline $\begin{array}{l}\text { Borreria schumannii (Standl. ex Bacigalupo) } \\
\text { E.L.Cabral \& Sobrado }\end{array}$ & SH 729 & epr & EBR & & & \\
\hline Borreria verticillata (L.) G. Mey & H 319 & ee & EBR & & $\mathrm{X}$ & \\
\hline Chomelia parvifolia (Standl.) Govaerts & Н 469 & arb & MAT & & & \\
\hline $\begin{array}{l}\text { Coccocypselum capitatum (Graham) C.B.Costa } \\
\& \text { Mamede }\end{array}$ & H 717 & epr & MAT & & $\mathrm{X}$ & \\
\hline Coccocypselum lanceolatum (Ruiz \& Pav.) Pers. & Н 344 & epr & EBR & & $\mathrm{X}$ & \\
\hline $\begin{array}{l}\text { Cordiera myrciifolia (K. Schum.) C.H.Perss. } \\
\& \text { Delprete }\end{array}$ & H 648 & árv & EBR & & $\mathrm{X}$ & \\
\hline Diodia saponariifolia (Cham. \& Schltdl.) K. Schum. & H 336 & epr & EBR & & & \\
\hline Manettia congesta (Vell.) K.Schum. & H 605 & epr & MAT & & & \\
\hline Posoqueria acutifolia Mart. & G 2076 & árv & BRA & & $\mathrm{X}$ & $4(\mathrm{fr}, \mathrm{s})$ \\
\hline Psychotria forsteronioides Müll.Arg. & H 715 & arb & EBR & & $\mathrm{X}$ & \\
\hline Psychotria leiocarpa Cham. \& Schltdl. & G 3250 & avt & BRA & & & \\
\hline Psychotria longipes Müll. Arg. & G 3244 & árv & BRA & & & \\
\hline Psychotria suterella Müll.Arg. & G 3242 & árv & EBR & & $\mathrm{X}$ & $4(s)$ \\
\hline Psychotria vellosiana Benth. & G 3193 & avt & BRA & & $\mathrm{X}$ & $4(\mathrm{~s})$ \\
\hline Rudgea jasminoides (Cham.) Müll. Arg. & G 3197 & avt & EBR & & $\mathrm{X}$ & \\
\hline \multicolumn{7}{|l|}{ RUTACEAE } \\
\hline Zanthoxylum fagara (L.) Sarg. & S 29 & árv & EBR & & & \\
\hline \multicolumn{7}{|l|}{ SALICACEAE } \\
\hline Casearia decandra Jacq. & S 46 & árv & EBR & & $\mathrm{X}$ & $4(\mathrm{fr}, \mathrm{s})$ \\
\hline Casearia obliqua Spreng. & H 327 & árv & BRA & & $\mathrm{X}$ & \\
\hline Casearia sylvestris $\mathrm{Sw}$. & Н 772 & árv & BRA & & $\mathrm{X}$ & 5 (fo), 7 (fo) \\
\hline
\end{tabular}


GARCIA, R.J.F.; HONDA, S.; FRIES, B.G. Estudo florístico de Mata Atlântica para soltura de bugio-ruivo, Alouatta clamitans

continuação - Tabela 1

continuation - Table 1

\begin{tabular}{|c|c|c|c|c|c|c|}
\hline $\begin{array}{l}\text { Grupo } \\
\text { Família } \\
\text { Espécie }\end{array}$ & $\begin{array}{l}\text { Voucher } \\
\text { de } \\
\text { coleta }\end{array}$ & hábito & $\begin{array}{l}\text { Distribuição } \\
\text { geográfica }\end{array}$ & $\begin{array}{l}\text { Estado de } \\
\text { Conservação }\end{array}$ & PEFI & alimento \\
\hline \multicolumn{7}{|l|}{ SANTALACEAE } \\
\hline Phoradendron crassifolium (Pohl ex DC.) Eichler & H 809 & hp & EBR & & $\mathrm{X}$ & \\
\hline \multicolumn{7}{|l|}{ SAPINDACEAE } \\
\hline Allophylus petiolulatus Radlk. & G 3443 & avt & EBR & & $\mathrm{X}$ & \\
\hline Cupania oblongifolia Mart. & Н 329 & árv & BRA & & $\mathrm{X}$ & \\
\hline Cupania vernalis Cambess. & H 783 & árv & EBR & & $\mathrm{X}$ & \\
\hline Matayba guianensis Aubl. & Н 339 & árv & EBR & & & \\
\hline Matayba juglandifolia (Cambess.) Radlk. & S 16A & árv & BRA & & $\mathrm{X}$ & \\
\hline Paullinia carpopoda Cambess. & H 710 & $\operatorname{tr}$ & BRA & & $\mathrm{X}$ & \\
\hline Paullinia trigonia Vell. & H 889 & $\operatorname{tr}$ & BRA & & $\mathrm{X}$ & \\
\hline Serjania communis Cambess. & H 788 & $\operatorname{tr}$ & EBR & & $\mathrm{X}$ & \\
\hline \multicolumn{7}{|l|}{ SAPOTACEAE } \\
\hline Diploon cuspidatum (Hoehne) Cronquist & Н 345 & árv & EBR & & $\mathrm{X}$ & $4(\mathrm{fr}, \mathrm{s})$ \\
\hline \multicolumn{7}{|l|}{ SCROPHULARIACEAE } \\
\hline Buddleja stachyoides Cham. \& Schltdl. & Н 697 & arb & EBR & & $\mathrm{X}$ & \\
\hline \multicolumn{7}{|l|}{ SOLANACEAE } \\
\hline Brunfelsia pauciflora Cham. \& Schltdl. & G 3238 & arb & MAT & & $\mathrm{X}$ & \\
\hline Capsicum mirabile Mart. & H 600 & arb & MAT & & & \\
\hline Cestrum bracteatum Link \& Otto & Н 381 & arb & EBR & & $X$ & \\
\hline Cestrum schlechtendalii G.Don & H 508 & avt & EBR & & $\mathrm{X}$ & \\
\hline Nicotiana langsdorffii Weinm. & Н 792 & ee & EBR & & $\mathrm{X}$ & \\
\hline Physalis pubescens $\mathrm{L}$. & G 2072 & ee & EBR & & $\mathrm{X}$ & \\
\hline Solanum bullatum Vell. & Н 782 & árv & MAT & & $\mathrm{X}$ & \\
\hline Solanum cinnamomeum Sendtn. & G 3191 & árv & MAT & & $\mathrm{X}$ & \\
\hline Solanum concinnum Schott ex Sendtn. & Н 665 & arb & MAT & & $\mathrm{X}$ & \\
\hline Solanum granulosoleprosum Dunal & Н 353 & arb & EBR & & $\mathrm{X}$ & \\
\hline Solanum inodorum Vell. & Н 721 & $\operatorname{tr}$ & MAT & & $\mathrm{X}$ & \\
\hline Solanum pseudoquina A.St.-Hil. & Н 598 & árv & EBR & & $\mathrm{X}$ & 7 (fo), 4 (s) \\
\hline Solanum rufescens Sendtn. & G 3195 & arb & MAT & & $\mathrm{X}$ & $4(s)$ \\
\hline Solanum swartzianum Roem. \& Schult. & Н 626 & avt & EBR & & $\mathrm{X}$ & \\
\hline Solanum wacketii Witasek & H 404 & arb & MAT & & & \\
\hline
\end{tabular}


continuação - Tabela 1

continuation - Table 1

\begin{tabular}{|c|c|c|c|c|c|c|}
\hline $\begin{array}{l}\text { Grupo } \\
\text { Família } \\
\text { Espécie }\end{array}$ & $\begin{array}{l}\text { Voucher } \\
\text { de } \\
\text { coleta }\end{array}$ & hábito & $\begin{array}{l}\text { Distribuição } \\
\text { geográfica }\end{array}$ & $\begin{array}{l}\text { Estado de } \\
\text { Conservação }\end{array}$ & PEFI & alimento \\
\hline \multicolumn{7}{|l|}{ SYMPLOCACEAE } \\
\hline Symplocos estrellensis Casar. & H 732 & árv & MAT & & & \\
\hline Symplocos sp1. [S. kleinii A.Bidá] & Н 660 & avt & ND & & & \\
\hline Symplocos sp2. & S 48 & avt & ND & & & \\
\hline \multicolumn{7}{|l|}{ THYMELAEACEAE } \\
\hline Daphnopsis fasciculata (Meisn.) Nevling & H 724 & avt & BRA & & $\mathrm{X}$ & \\
\hline \multicolumn{7}{|l|}{ URTICACEAE } \\
\hline Cecropia glaziovii Snethl. & H 817 & árv & MAT & & $\mathrm{X}$ & \\
\hline Cecropia hololeuca Miq. & Н 320 & árv & BRA & NT & $\mathrm{X}$ & 3 (fr) \\
\hline Urera baccifera (L.) Gaudich. ex Wedd. & G 2070 & ee & EBR & & $\mathrm{X}$ & \\
\hline \multicolumn{7}{|l|}{ VALERIANACEAE } \\
\hline Valeriana scandens $\mathrm{L}$. & H 810 & $\operatorname{tr}$ & EBR & & $\mathrm{X}$ & \\
\hline \multicolumn{7}{|l|}{ VERBENACEAE } \\
\hline Lantana camara $\mathrm{L}$. & H 700 & arb & EBR & & $\mathrm{X}$ & \\
\hline \multicolumn{7}{|l|}{ VIOLACEAE } \\
\hline Anchietea pyrifolia (Mart.) G.Don & G 3210 & $\operatorname{tr}$ & EBR & & $\mathrm{X}$ & \\
\hline
\end{tabular}

Quanto à distribuição dessas espécies nos diversos componentes, foram registradas 132 espécies $(52,6 \%)$ no componente arbóreo-arbustivo (árvores, arbustos, arvoretas, bambus, palmeiras e fetos arborescentes), 66 espécies (26,3\%) no componente herbáceo (ervas) e 53 espécies $(21,1 \%)$ no componente epifítico (epífitas, trepadeiras e hemiparasitas).

Dentre as 185 espécies dos componentes arbóreo-arbustivo e epifítico, que constituem o hábitat preferencial dos bugios-ruivos, 33 (17,8\%) espécies registradas no presente levantamento constituem-se em fontes alimentares já documentadas para a espécie (Tabela 1). Ressalva-se que este número pode ser maior, uma vez que os dados disponíveis na literatura contêm identificações incompletas; por exemplo, dentre as referências consultadas, 14 espécies identificadas apenas até o nível genérico têm representantes na área de estudo. Considerando-se a possível similaridade química de espécies de um gênero, pode-se supor que este número seria ainda maior.
Ressalta-se que dentre essas 33 espécies já referidas como consumidas por bugios-ruivos, todas apresentam distribuição ampla, sendo 19 $(57,6 \%)$ brasileiras e extrabrasileiras, 8 brasileiras não restritas à Mata Atlântica $(24,2 \%)$ e 6 (18,2\%) restritas à Mata Atlântica. Estes dados podem tanto indicar falta de estudos sobre hábitos alimentares como também uma preferência da espécie por plantas de ampla distribuição, que já estariam melhor assimiladas ao repertório de experiências dos bugios-ruivos. A partir do monitoramento dos indivíduos reintroduzidos no município de São Paulo pretende-se verificar a utilização de outras fontes de alimento (B.G.Fries, comunicação pessoal).

Além das espécies vegetais consumidas, constata-se que $75 \%$ das espécies de bromélias registradas na área de estudo podem acumular água em suas rosetas foliares (bromélias-tanque), propiciando condições para a dessedentação dos animais no dossel da mata, diminuindo a necessidade de os animais descerem ao solo em busca de água e, portanto, diminuindo possíveis acidentes (atropelamentos, caça, ataque de cães). 
No município de São Paulo, além da presente área de estudo, apenas o Parque Estadual das Fontes do Ipiranga - PEFI tem registro de todos os componentes da vegetação (monografias segundo Melhem et al., 1981) e apresenta registros de bugios-ruivos, em populações bem estabelecidas. Dentre as 1.173 espécies ali registradas (dados normalizados do banco de dados do Herbário Municipal), $71,6 \%$ são dos componentes arbóreo-arbustivo e epifítico, o que está proporcionalmente próximo ao observado para a presente área de estudo $(73,7 \%)$. Para este grupo de espécies do PEFI, 9,4\% apresentam registro de consumo por bugios-ruivos, enquanto na presente área de estudo foram registradas 17,8\%. Além disso, $71,4 \%$ das fanerógamas da presente área de estudo também ocorrem no PEFI (Tabela 1), indicando que a área de estudo apresenta potencial florístico que suporta a reintrodução.

A presença dos bugios-ruivos no fragmento florestal poderá contribuir para a dispersão de sementes no local, tendo em vista que 14 espécies (42,2\% das 33 registradas como consumidas) foram assinaladas na literatura com consumo de frutos, 12 espécies $(36,4 \%)$ foram registradas com consumo de sementes, o que poderia causar impacto negativo a estas espécies, inclusive para a única registrada como vulnerável, Araucaria angustifolia (Mamede et al., 2007).

Dadas as condições de variabilidade temporal na disponibilidade dos recursos alimentares, é possível que as 15 espécies $(45,4 \%$ das 33 do repertório conhecido) que são procuradas para consumo de folhas tenham papel importante na manutenção desses animais. Entre as árvores, cabe ressaltar o potencial de Alchornea sidifolia, Clethra scabra e Piptocarpha spp., pela abundância, e Hymenaea courbaril e Cariniana estrellensis, pelo volume da copa. No grupo de espécies procuradas pelas folhas, apenas Cedrela fissilis é caducifólia no inverno.

Galetti et al. (1994) registraram a importância das folhas de trepadeiras na dieta alimentar dos bugios-ruivos, especialmente de Bignoniaceae, Malpighiaceae e Sapindaceae. Para a área de estudo, foram registradas seis espécies de trepadeiras destas famílias, além de 23 espécies de outras famílias, embora nenhuma delas tenha o consumo de folhas registrado ainda.

A área de estudo apresentou duas espécies vulneráveis (VU) e quatro quase ameaçadas (NT) (Mamede et al., 2007), constituindo-se em importante local para preservação das mesmas e possível inclusão em programas de coleta de sementes.

\section{CONCLUSÕES}

A área selecionada para o levantamento florístico apresentou elevada riqueza e diversidade de componentes, incluindo recursos alimentares importantes para a manutenção dos bugios-ruivos reintroduzidos.

Como os bugios-ruivos também se alimentam de frutos, podem assim contribuir para a dispersão de sementes na mata, favorecendo o enriquecimento da vegetação local. A escolha da área a partir das características fisionômicas foi satisfatória, visto que pelo menos $17,8 \%$ das espécies dos componentes arbóreo e epifítico podem ser utilizadas pelos bugios-ruivos como recurso alimentar.

A presença de bromélias-tanque foi observada como fator importante para a população (recurso água). Assim, faz-se necessário incrementar programas para estudos visando reintroduzir espécies de bromélias, a partir da coleção viva de DEPAVE-2.

Alguns desdobramentos do presente estudo, que poderão propiciar subsídios a novas solturas, são os testes com novas opções de alimento que podem ser oferecidas no período em que os animais estão em cativeiro (pré-soltura), e plantios de enriquecimento de matas no município a fim de favorecer a conservação da espécie-alvo.

O levantamento realizado apresenta informações de uma área ainda pouco estudada do município, podendo contribuir também para outras ações de conservação ambiental em escala regional. 


\section{AGRADECIMENTOS}

Os autores agradecem a Marcos K. Vasconcellos pela produção da figura; aos estagiários e funcionários do Herbário Municipal e da Divisão de Fauna que colaboraram em atividades de campo e laboratório e ao Sr. Nazir Haddad.

\section{REFERÊNCIAS BIBLIOGRÁFICAS}

BAKER, L.R. (Ed.). IUCN/SSC Re-introduction Specialist Group: guidelines for nonhuman primate re-introductions. Re-introduction News, n. 21, p. 29-57, 2002.

CARVALHO, C.T. Acerca da alimentação dos bugios (Mammalia, Cebidae). Silvicultura em São Paulo, v. 9, p. 53-56, 1975.

CHITOLINA, O.P.; SANDER, M. Contribuição ao conhecimento de Alouatta guariba clamitans Cabrera, 1940 em habitat natural no Rio Grande do Sul. Iheringia, Série Zoologia, v. 59, p. 37-44, 1981.

FIDALGO, O.; BONONI, V.L.R. (Coord.). Técnicas de coleta, preservação e herborização de material botânico. São Paulo: Instituto de Botânica, 1989. 62 p.

FORZZA, R.C. et al. Lista de espécies da flora do Brasil 2013. Jardim Botânico do Rio de Janeiro. Disponível em: <http://floradobrasil.jbrj.gov.br/>. Acesso em: 23 dez. 2013.

FRIES, B.G. et al. Adaptação pré-soltura e soltura de bugio-ruivo Alouatta guariba clamitans (Cabrera, 1940), (Primates, Atelidae), em áreas de mata atlântica no município de São Paulo. In: MELO, F.R.; MOURTHÉ, Í. (Ed.) A Primatologia no Brasil. Belo Horizonte: Sociedade Brasileira de Primatologia, 2012. v. 11, p. 211-227.

GALETTI, M.; PEDRONI, F.; MORELLATO, L.P.C. Diet of the brown monkey Alouatta fusca in a forest fragment in southern Brazil. Mammalia, v. 58, n. 1, p. 111-118, 1994.

GREGORIN, R. Taxonomia e variação geográfica das espécies do gênero Alouatta Lacépède (Primates, Atelidae) no Brasil. Revista Brasileira de Zoologia, v. 23, n. 1, p. 64-144, 2006.
INSTITUTO BRASILEIRO DO MEIO AMBIENTE E DOS RECURSOS NATURAIS RENOVÁVEIS IBAMA. Instrução Normativa $\mathrm{n}^{\circ} 179$, de 25 de junho 2008. Defini as diretrizes e procedimentos para destinação dos animais da fauna silvestre nativa e exótica apreendidos, resgatados ou entregues espontaneamente às autoridades competentes. Diário Oficial da União, n. 121, 26 jun. 2008. Seção 1, p. 60.

INTERNATIONAL UNION FOR CONSERVATION OF NATURE - IUCN. IUCN red list of threatened species. Versão 2010. Disponível em: <www.iucnredlist.org>. Acesso em: 13 ago. 2013.

KUHLMANN, M. Adenda alimentar dos bugios. Silvicultura em São Paulo, v. 9, p. 57-62, 1975.

MAMEDE, M.C.H. et al. Livro vermelho das espécies vegetais ameaçadas do Estado de São Paulo. São Paulo: Instituto de Botânica, 2007. $165 \mathrm{p}$.

MELHEM, T.S. et al. Planejamento para a elaboração da "Flora Fanerogâmica da Reserva do Parque Estadual das Fontes do Ipiranga (São Paulo, Brasil). Hoehnea, v. 9, p. 63-74, 1981.

MIRANDA, J.M.D.; PASSOS, F.C. Hábito alimentar de Alouatta guariba (Humboldt) (Primates, Atelidae) em Floresta de Araucária, Paraná, Brasil. Revista Brasileira de Zoologia, v. 21, n. 4, p. 821-826, 2004.

SÃO PAULO (Estado). Decreto Estadual no 60.133, de 7 de fevereiro de 2014. Declara as espécies da fauna silvestre ameaçadas de extinção, as quase ameaçadas e as deficientes de dados para avaliação no Estado de São Paulo e dá providências correlatas. Diário Oficial do Estado de São Paulo, Poder Executivo, v. 124, n. 27, 8 fev. 2014. Seção I, p. 25-32.

SÃO PAULO (Município). Resolução $n^{\circ}$ 19/ CONFEMA/2006, de 6 de junho de 2006. Dispõe sobre a aprovação do projeto candidato a recursos do FEMA, apresentado pela Secretaria Municipal do Verde e do Meio Ambiente. Diário Oficial da Cidade de São Paulo, v. 51, n. 108, 9 jun. 2006, p. 27. 
GARCIA, R.J.F.; HONDA, S.; FRIES, B.G. Estudo florístico de Mata Atlântica para soltura de bugio-ruivo, Alouatta clamitans

SILVA JÚNIOR, E.C. A preliminary survey of brown howler monkeys (Alouatta fusca) at the Cantareira Reserve (São Paulo, Brazil). Revista Brasileira de Biologia, v. 41, n. 4, p. 897-909, 1980.

VASCONCELLOS, L.E.M.; AGUIAR, O.T. A alimentação de Alouatta fusca Geof. (Primates, Cebidae). In: CONGRESSO NACIONAL SOBRE ESSÊNCIAS NATIVAS, 1982, Campos do Jordão. Anais... São Paulo: UNIPRESS, 1982. p. 1727-1730. (Silvicultura em São Paulo, v.16-A, pt. 3, 1982). 